\title{
Baseline LV ejection fraction by cardiac magnetic resonance and 2D echocardiography after ST-elevation myocardial infarction - influence of infarct location and prognostic impact
}

\author{
Johannes P. Schwaiger ${ }^{1}$. Sebastian J. Reinstadler ${ }^{2} \cdot$ Christina Tiller $^{2} \cdot$ Magdalena Holzknecht $^{2}$ - Martin Reindl ${ }^{2}$. \\ Agnes Mayr ${ }^{3} \cdot$ Ivo Graziadei $^{1} \cdot$ Silvana Müller $^{2} \cdot$ Bernhard Metzler $^{2} \cdot$ Gert Klug $^{2}$
}

Received: 15 February 2019 / Revised: 22 May 2019 / Accepted: 10 June 2019/Published online: 19 August 2019

(C) The Author(s) 2019

\begin{abstract}
Objectives The comparability of left ventricular ejection fraction (LVEF) measurements by cardiac magnetic resonance (CMR) and 2D echocardiography (2DE) early after ST-elevation myocardial infarction (STEMI) remains unclear.

Methods In this study, LVEF measured by CMR and 2DE (Simpson's method) were compared in 221 patients after STEMI treated by primary percutaneous coronary intervention. 2DE image quality was systematically assessed and studies reported by an accredited examiner. Intermodality agreement was assessed by the Bland-Altman method. Major adverse cardiac events (MACE) were defined as the composite of death, myocardial infarction or hospitalisation for heart failure. Patients were followed up for a median of 40.9 months (IQR 28.1-56).

Results After non-anterior STEMI, LVEF measurements by 2DE (single and biplane) were consistently underestimated in comparison to CMR (CMR 55.7 $\pm 9.5 \%$ vs. $2 \mathrm{DE}-4 \mathrm{CV} 49 \pm 8.2 \%$ ( $p=0.06$ ), 2DE-2CV $52 \pm 8 \%(p<0.001)$, 2DE-biplane $53.5 \pm 7.1 \%(p=0.01))$. After anterior STEMI, there was no significant difference in LVEF measurements by $2 \mathrm{DE}$ and CMR with acceptable limits of agreement (CMR $49 \pm 11 \%$ vs. $2 \mathrm{DE}-4 \mathrm{CV} 49 \pm 8.2 \%(p=0.8), 2 \mathrm{DE}-2 \mathrm{CV} 49 \pm 9.2 \%(p=0.9)$, 2DEbiplane $49.6 \pm 8 \%(p=0.5))$. In total, $15 \%$ of patients experienced a MACE during follow-up. In multivariate Cox regression analysis, reduced LVEF $(<52 \%)$ as assessed by either 2DE or CMR was predictive of MACE (2DE HR $=2.57$ (95\% CI 1.1-6.2), $p=0.036 ;$ CMR HR $=2.51(95 \%$ CI 1.1-5.7), $p=0.028)$.

Conclusions At baseline after non-anterior STEMI, 2D echocardiography significantly underestimated LVEF in comparison to CMR, whereas after anterior infarction, measurements were within acceptable limits of agreement. Both imaging modalities offered similar prognostic values when a reduced LVEF $<52 \%$ was applied.

Key Points

- After non-anterior STEMI, 2D-echocardiography significantly underestimated LVEF compared with cardiac MRI

- An ejection fraction of $<52 \%$ in the acute post-infarct period by both $2 D$ echocardiography and CMR offered similar prognostic values
\end{abstract}

Keywords Anterior wall myocardial infarction $\cdot$ Magnetic resonance imaging $\cdot$ Cine $\cdot$ Echocardiography $\cdot$ ST-elevation myocardial infarction

Gert Klug

gert.klug@tirol-kliniken.at

1 Department of Internal Medicine, Academic Teaching Hospital Hall in Tirol, Innsbruck, Austria

2 University Clinic of Internal Medicine III, Cardiology and Angiology, Medical University of Innsbruck, Anichstrasse 35, 6020 Innsbruck, Austria

3 Department of Radiology, Medical University of Innsbruck, Innsbruck, Austria

\begin{tabular}{ll}
\multicolumn{2}{l}{ Abbreviation } \\
2CV & Two-chamber view \\
2DE & 2D echocardiography \\
4CV & Four-chamber view \\
CMR & Cardiac magnetic resonance \\
DENSE & Displacement encoding with stimulated echoes \\
HR & Hazard ratio \\
IQR & Interquartile range \\
LGE & Late gadolinium enhancement \\
LVEF & Left ventricular ejection fraction
\end{tabular}


MACE Major cardiac event

PCI Percutaneous coronary intervention

SD Standard deviation

STEMI ST-elevation myocardial infarction

\section{Introduction}

Left ventricular ejection fraction (LVEF) is of major prognostic importance in many cardiac diseases, in particular after STsegment elevation myocardial infarction (STEMI) [1-4]. Guidelines recommend transthoracic echocardiography before discharge after STEMI to assess for infarct size and resting LV function, identifying patients at high risk for worse outcome [5]. In addition, it provides important information about diastolic function in the acute phase [6]. However, there are significant limitations due to often inaccurate discrimination of the endocardial border $[7,8]$ and the exam can be particularly cumbersome in acute cardiac disease due to arrhythmia, stress or hypercontractility of adjacent segments [9]. In addition, its accuracy is dependent on the examiner's experience and training and therefore offers only moderate reproducibility $[10,11]$.

For these reasons, cardiac magnetic resonance (CMR) imaging has evolved into the gold-standard imaging method of systolic function [12-14]; however, few studies have compared ejection fraction measurements determined by $2 \mathrm{DE}$ and CMR in the immediate post-infarct period. In one small study, LVEF was poorly correlated and overestimated by $2 \mathrm{DE}$ [15], whereas in another, larger study, LVEF was underestimated by 2DE [16]. None of these studies has systematically assessed 2DE quality or has taken infarct location into consideration.

The aim of this study was (a) to compare LVEF measurements by CMR and 2DE at baseline after STEMI, (b) to examine influence of infarct location on 2DE LVEF measurements and (c) to assess the prognostic comparability of $2 \mathrm{DE}$ and CMR in the immediate post-infarct period. Particular emphasis was put on retrospective assessment of 2DE image quality; all studies were reported by an individual with the appropriate experience and accreditation.

\section{Methods}

\section{Study population}

The patients in this study were participants in a prospective analysis enrolling patients who underwent CMR after acute STEMI treated with primary percutaneous coronary intervention (PCI) $[4,17,18]$. Inclusion criteria were the diagnosis of first STEMI according to the redefined ESC/ACC committee criteria [19] and primary PCI within $24 \mathrm{~h}$ of symptom onset.
Exclusion criteria were renal dysfunction with an estimated glomerular filtration rate $<30 \mathrm{ml} / \mathrm{min} / 1.73 \mathrm{~m}^{2}$, Killip class $>2$ at time of CMR acquisition and contraindications for CMR.

Demographic data and clinical profile of patients were acquired with the help of a standardised questionnaire during hospitalisation for STEMI. Blood samples were collected as previously reported [20]. The study complies with the Declaration of Helsinki and the local ethics committee approved the study protocol. Written informed consent was obtained from all participants.

\section{Echocardiography}

Echocardiography was performed in routine clinical practice in the Echocardiography Department of the University Hospital Innsbruck according to current guidelines [21]. Native exams were reloaded from the PACS and analysed twice by a single consultant cardiologist (JPS) at a time interval of 2 months and measurements averaged. Measurements were used to calculate intraobserver variabilities. JPS has significant experience and is certified in transthoracic and transoesophageal echocardiography by the European Society of Cardiology. JPS was blinded to CMR and clinical results. Echocardiograms were analysed on the IMAGE-COM® Software of TOMTEC Imaging Systems.

Determination of end-diastolic and end-systolic volumes was performed on four-chamber view (4CV) and twochamber view (2CV) according to the Simpson method. Similar to others, we have used a 5-point scale to assess image quality [22]. Each 4CV and 2CV was assessed separately to increase accuracy of quality assessment. Therefore, a maximum of 10 points was allowed if there was complete endocardial definition and typical configuration (e.g. no foreshortening in $4 \mathrm{CV}$ or posterolateral papillary muscle visible in $2 \mathrm{CV}$ ) in both views. Four points were allowed for each view when picture quality was mildly reduced, 3 when moderate and 2 when moderately reduced and 1 point if image quality was of borderline quality. Interobserver variabilities were calculated using 50 subjects which were selected evenly across the spectrum of 2D echocardiography quality by a second observer accredited in transthoracic and transoesophageal echocardiography by the European Society of Cardiology (GK). Observer variability analyses were performed without knowledge of patient identity or previous results.

\section{CMR protocol and image evaluation}

All scans were performed on a 1.5-Tesla Magnetom AVANTO scanner (Siemens). Briefly, cine CMR images in short-axis were acquired using breath-hold, retrospective ECGtriggered TrueFISP bright-blood sequences. Evaluation of images was performed using a standard software (ARGUS, Siemens). End-diastolic and end-systolic volumes as well as 
stroke volume and LVEF were obtained from manual delineation of the endocardial borders. The most basal slice in endsystolic views was discarded if myocardium was not present in less than a half of the ventricular circumference. Trabeculation and papillary muscles were included into the LV volume. Late gadolinium enhancement (LGE) images were acquired by using an ECG-triggered phase-sensitive inversion recovery single-shot TrueFISP sequence with consecutive short-axis slices as described in detail previously [23-26]. Infarct localisation was assessed LGE CMR. Cardiac magnetic resonance images were acquired under the supervision of a EuroCMR level II-certified radiologist (AM) with a long experience in CMR (> 10 years). Analysis of CMR cine volumetric data was performed by individuals trained in volumetric dataset analysis and reviewed by at least level I-certified individuals with a long experience in CMR (>10 years GK, AM; > 5 years SJR).

\section{Clinical follow-up}

A LVEF of $<52 \%$ was chosen as the cut-off for reduced LVEF in both image modalities because it represented both the mean and median in the 2DE population and it corresponds to the lower limit of normal in current echocardiography guidelines for male patients [21], which fits the male predominance in our study. To ensure intermodality comparability, the same cut-off was chosen for CMR.

Patients were followed for major adverse cardiac events (MACE), defined as a composite of death, myocardial reinfarction and new congestive heart failure as previously described [27]. Time to MACE was defined as time from PCI to the first end-point.

\section{Statistical analysis}

Statistical analysis was performed using SPSS for Windows, release 22.0.0.1 (IBM). The Shapiro-Wilk test was used to test for normal distribution. Normally distributed continuous data are presented as mean \pm standard deviation (SD) and comparisons were performed by using paired $t$ test. Nonnormally distributed continuous data are presented as median with interquartile range (IQR) and comparisons were performed with non-parametric Wilkoxon signed-rank test. Comparisons of normally distributed continuous data in patients with or without MACE were performed using unpaired $t$ test or Mann-Whitney $U$ Test in the case of non-normally distributed data. Correlation of variables was performed using Pearson correlation coefficient. $P$ values $<0.05$ were considered to indicate statistical significance. Intermodality agreement was studied using the Bland-Altman method, whereby the mean difference was presented as the bias and $95 \%$ limits of agreement around the bias expressed as the mean difference \pm 1.96 SDs. Interobserver and intraobserver variations of
LVEF by 2DE were computed as the root of the mean squared differences between corresponding observations, divided by the number of observations. Intra- and interobserver correlations were calculated using intraclass correlation coefficient.

Kaplan-Meier survival curves were used to describe the cumulative incidence of event-free survival over time, and log-rank test was used to test for differences. Multivariate Cox regression analysis, adjusted for sex and age, was applied to evaluate the effect of LVEF on MACE.

\section{Results}

\section{Study population}

During the study period, 323 STEMI patients were enrolled into the CMR database. In 271 of these, transthoracic echocardiograms were performed in addition to CMR and pictures archived in the hospital's PACS. In 221 of these, appropriate $4 \mathrm{CV}$ and $2 \mathrm{CV}$ were available and analysed. One patient was excluded due to severe breathing artefacts in CMR. Echocardiograms were performed a median of 3 (IQR 2-5), and CMR a median of 2.6 days after STEMI (IQR 2-4). The mean difference between exams was $0.4 \pm 1.8$ days. Mean heart rate during MR was $74 \pm 16$; three patients had heart rates $>110$ beats $/ \mathrm{min}$.

The mean age of the study participants was $58 \pm 11$ years; 34 patients $(15.4 \%)$ were female. Detailed patient characteristics on admission are shown in Table 1.

\section{Echocardiography image quality}

Echocardiography image quality was evenly distributed, with the majority of echocardiograms being of moderate quality (5-7 points) (Fig. 1). Echocardiograms of lower quality $(\leq 4$ points; $n=39 ; 17.7 \%$ ) were excluded, leaving 181 patients for subsequent analysis.

\section{Comparison of LVEF measurements and volumes}

LVEF measurements were moderately correlated $(r=0.589$; $p<0.0001)$ and not statistically different from each other (CMR $53 \pm 11 \%$ vs. 2 DE $52 \pm 8 \%$; $p=0.16$ ). Only a minority of patients (12) demonstrated a severely reduced LVEF of $<35 \%$ during CMR. Figure 2 (panel a) shows the BlandAltman plot demonstrating a small mean difference though relatively wide limits of agreement $(-17.6$ to $+19 \%)$.

\section{Influence of infarct location}

Figure 2 (panel b) demonstrates differences between LVEF measurements in anterior and non-anterior infarctions by $2 \mathrm{DE}$ and CMR. After anterior infarction, both 
Table 1 Patient characteristics $(n=221)$

\begin{tabular}{lc}
\hline Age (years) & $58 \pm 11$ \\
Sex (m/f) & $187 / 34$ \\
Body mass index & $26 \pm 3$ \\
Diabetes (\%) & 13 \\
Smoking (\%) & 50 \\
Hypertension (\%) & 58 \\
Total cholesterol (mg/dl) & $93 \pm 43$ \\
Hyperlipidaemia (\%) & $67 \%$ \\
Creatinine (mg/dl) & $0.94 \pm 0.2$ \\
CK max (U/l) & $2110 ; \mathrm{IQR} 1159-3473$ \\
Location of infarction (anterior/non-anterior) & $99 / 122$ \\
Culprit lesion & \\
RCA & $41 \%$ \\
LAD & $45 \%$ \\
CX & $13 \%$ \\
RI & $1 \%$ \\
Time from symptom onset to PCI (min) & $210 ; \mathrm{IQR} 139-396$ \\
TIMI flow pre/post (0, I, II, III) (\%) & \\
0 & $71 / 3.8$ \\
I & $14 / 0.5$ \\
II & $13.6 / 13.1$ \\
III & $1.3 / 82.6$ \\
\hline
\end{tabular}

$C K$, creatine kinase; $I Q R$, interquartile range; $R C A$, right coronary artery; $L A D$, left anterior descending artery; $C X$, circumflex artery; $R I$, ramus intermedius; TIMI, thrombolysis in myocardial infarction

single- (4CV and 2CV) and biplane LVEF measurements were not statistically different from measurements by CMR (CMR $49 \pm 11 \%$ vs. $2 \mathrm{DE}-4 \mathrm{CV} 49 \pm 8.2 \% \quad(p=0.8)$,

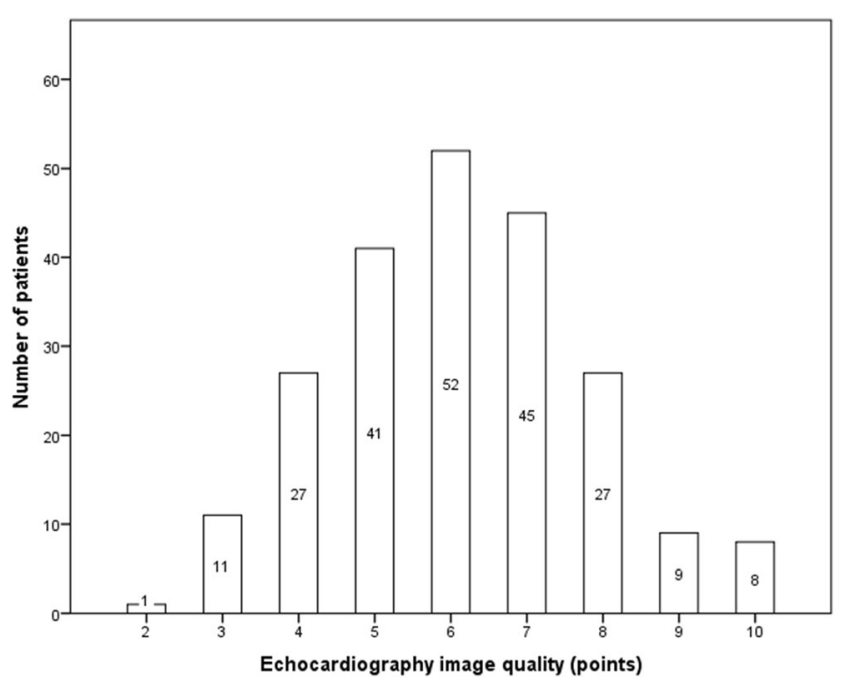

Fig. $12 \mathrm{D}$ echocardiography image quality. Figure demonstrating even distribution of 2D echocardiography image quality. Each $4 \mathrm{CV}$ and $2 \mathrm{CV}$ was assessed on a 5-point scale allowing a maximum of 10 points. Echocardiograms of lower quality ( $\leq 4$ points) were excluded from subsequent analysis. 4CV (four-chamber view); $2 \mathrm{CV}$ (two-chamber view)
2DE-2CV $49 \pm 9.2 \%(p=0.9)$, 2DE-biplane $49.6 \pm 8 \%$ $(p=0.5))$.

After non-anterior infarction, all 2DE measurements underestimated LVEF (CMR $55.7 \pm 9.5 \%$ vs. 2DE-biplane $53.5 \pm 7.1 \%(p=0.01) ; 2 \mathrm{DE}-4 \mathrm{CV} 54 \pm 8.2 \%(p=0.06)$; $2 \mathrm{DE}-2 \mathrm{CV} 52 \pm 8 \%(p<0.001))$.

2DE significantly underestimated end-diastolic $(130 \pm 29$ vs. $149 \pm 34 \mathrm{ml} ; p<0.01)$ and end-systolic $(64 \pm 20$ vs. $71 \pm$ $27 \mathrm{ml} ; p<0.01)$ volumes.

\section{Intra- and interobserver variabilities}

The mean intraobserver difference of LVEF measured by 2DE was $1.6 \pm 5.8 \%$; mean intraobserver variability was $3.1 \pm 3 \%$. The intraclass correlation coefficient was 0.844 (KI $0.78-$ $0.89 ; p<0.001)$. Mean interobserver difference was $0.32 \pm$ $7.9 \%$, mean variability was $4.6 \pm 3.1 \%$ and intraclass correlation coefficient was 0.801 (KI $0.65-0.88 ; p<0.001$ ). Intraand interobserver variabilities of LVEF measurements by our CMR lab were recently published elsewhere [28].

\section{Clinical follow-up}

In 161 patients (89\%), follow-up was completed, and 24 patients experienced a MACE after a median follow-up of 41 months (IQR 28.1-56). In total, $40.3 \%$ of patients were classified as reduced LVEF with CMR compared with 52\% when assessed with 2DE.

In univariate analysis, patients with MACE demonstrated significantly lower LVEF in both imaging modalities when compared with patients without MACE (Table 2). Figure 3 demonstrates Kaplan-Meier curves for each imaging modality showing significantly lower event-free survival in patients with reduced LVEF of $<52 \%$ (2DE $p=0.03$; CMR $p=0.025$; log-rank).

In multivariate Cox regression analysis, adjusted for sex and age, reduced LVEF $(<52 \%)$ as assessed by either $2 \mathrm{DE}$ or CMR was similarly predictive of MACE (2DE HR= 2.57 (95\% CI 1.1-6.2), $p=0.036$; CMR HR 2.51 (95\% CI 1.1-5.7), $p=0.028)$.

\section{Discussion}

The aim of the present study was (a) to compare LVEF measurements by $2 \mathrm{D}$ echocardiography to the gold-standard CMR at baseline after STEMI, (b) to examine the influence of infarct location on LVEF comparisons and (c) to assess the prognostic value of reduced LVEF at baseline as assessed by both imaging modalities over a follow-up period of 41 months. As 2DE largely remains an operator-dependent technique both in data acquisition and reporting, we have put particular emphasis on retrospective assessment of $2 \mathrm{DE}$ image quality and 


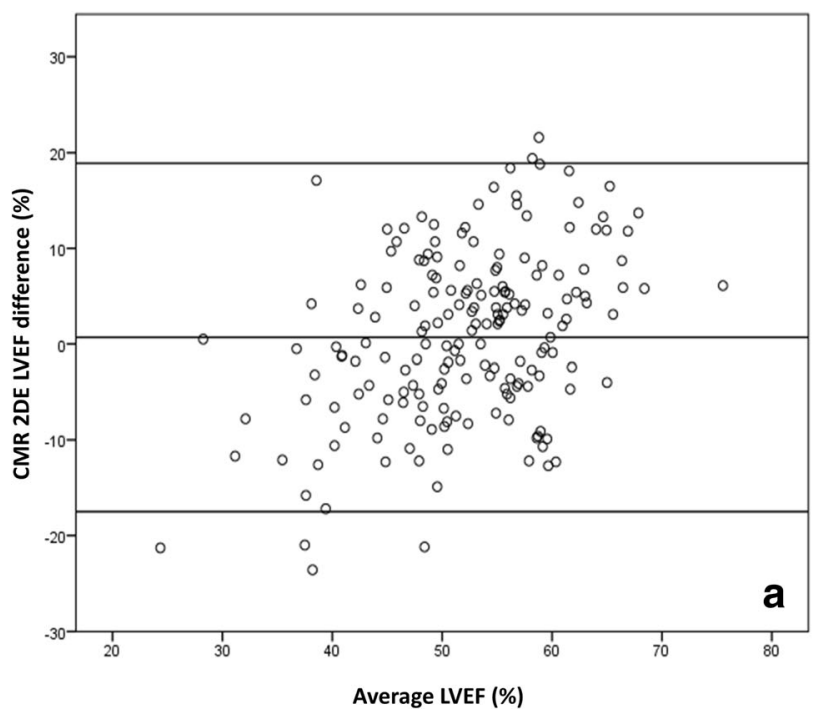

Fig. 2 LVEF comparisons CMR and 2DE. Panel a Bland-Altman plot of LVEF by 2DE and CMR. Bland-Altman diagram of ejection fraction (\%) demonstrating mean difference (middle line) and the limits of agreement (upper and lower lines). Panel $\mathbf{b}$ Comparison of LVEF measurements by 2DE (single and biplane) and CMR after non-anterior and anterior STEMI. Boxplot demonstrating better comparability of single- and biplane 2DE LVEF measurements with CMR measurements after anterior

all studies were reported by an individual with the appropriate experience and accreditation.

Although LVEF measurements at baseline by CMR and 2DE were not statistically different, limits of agreement were relatively wide, a phenomenon demonstrated in previous studies [29]. We demonstrated however a significant influence of infarct location on LVEF measurements by $2 \mathrm{DE}$. Whereas after anterior infarction both single- and biplane 2DE measurements appeared comparable with measurements by CMR, after non-anterior infarction, LVEF was significantly underestimated in both single- and biplane measurements.

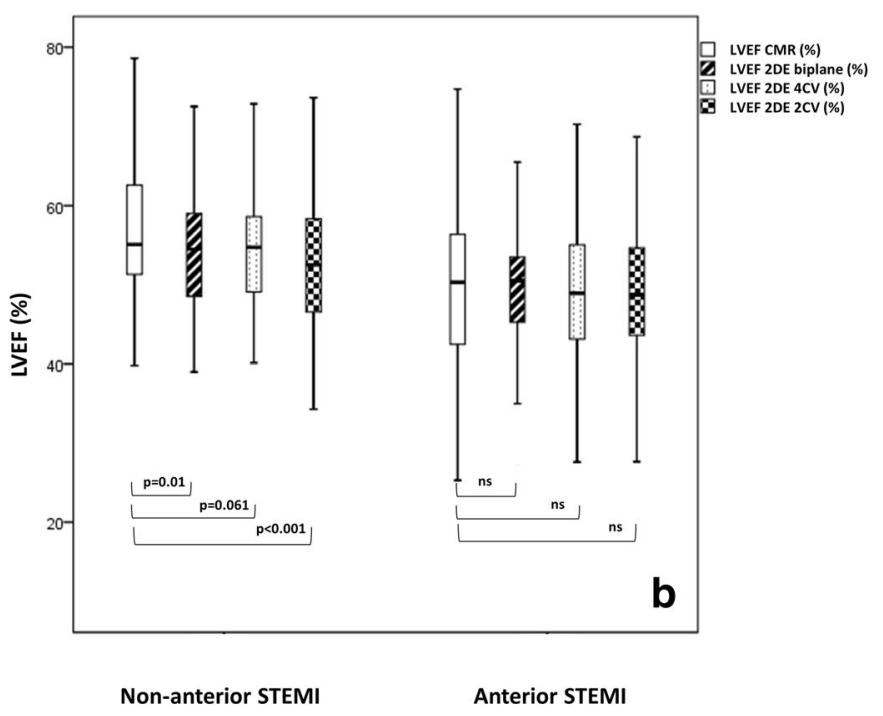

STEMI. Middle line in boxplot represents the median; whiskers represent 95\% CI. Comparison of means performed using paired $t$ test (significance level adjusted for multiplicity; $p=0.017)$. 2DE (2D echocardiography); $2 \mathrm{CV}$ (two-chamber view); 4CV (four-chamber view); CMR (cardiac magnetic resonance); LVEF (left ventricular ejection fraction); STEMI (ST-elevation myocardial infarction)

Two studies are available which have performed CMR and 2DE shortly after STEMI. A large group of 278 patients was examined and followed up by Waha et al; however, a direct comparison of LVEF was not reported, although measurements by $2 \mathrm{DE}$ appeared to be significantly underestimated with an absolute underestimation of $7 \%$ by $2 \mathrm{DE}$ [16]. Nowosielski et al have examined 52 STEMI patients and have found poor correlation of LVEF measurements demonstrating a large difference in the other direction [15]. Both studies did not report on 2DE image quality or infarct location.
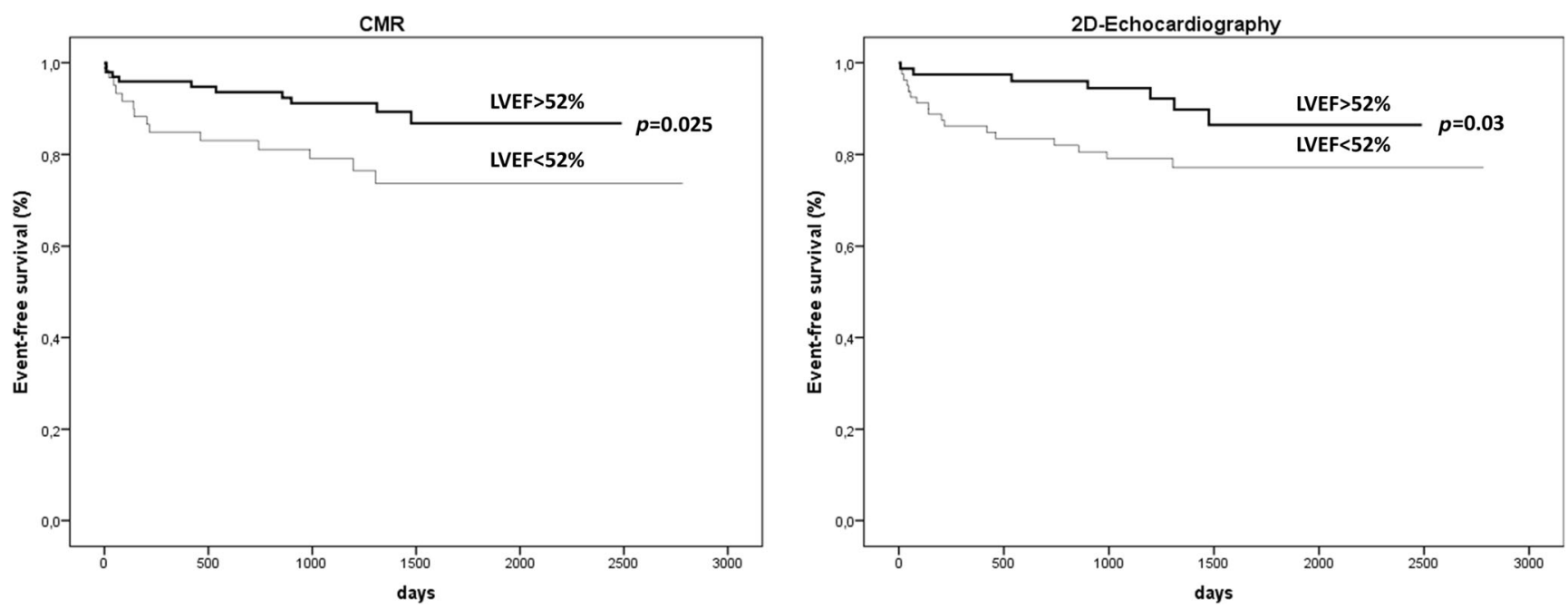

Fig. 3 Kaplan-Meier curves for event-free survival. Kaplan-Meier curves displaying event-free survival in relation to preserved versus reduced LVEF as assessed with 2DE and CMR. CMR (cardiac magnetic resonance); LVEF (left ventricular ejection fraction) 
Table 2 Selected baseline characteristics in patients with and without MACE during follow-up

\begin{tabular}{lccl}
\hline & MACE, $n=24$ & No MACE, $n=138$ & $p$ value \\
\hline LVEF CMR (\%) & $47 \pm 13$ & $54 \pm 10$ & $\mathbf{0 . 0 0 5}$ \\
EDV CMR (ml) & $155 \pm 40$ & $147 \pm 31$ & 0.45 \\
ESV CMR (ml) & $84 \pm 36$ & $68 \pm 24$ & $0.07 *$ \\
LVEF 2DE (\%) & $49 \pm 8$ & $52 \pm 7$ & $\mathbf{0 . 0 2}$ \\
EDV 2DE (ml) & $141 \pm 39$ & $128 \pm 28$ & 0.21 \\
ESV 2DE (ml) & $75 \pm 28$ & $62 \pm 18$ & $\mathbf{0 . 0 2}$ \\
\hline
\end{tabular}

$p$ values $<0.05$ are printed in bold

$C M R$, cardiac magnetic resonance; $E D V$, end-diastolic volume; $E S V$, endsystolic volume; $L V E F$, left ventricular ejection fraction; $M A C E$, major adverse cardiac event; 2DE, 2D echocardiography

*Mann-Whitney $U$ test

More data are available after a previous infarction. The largest study was performed in 150 patients 3 months after STEMI [29]. Very similar to our results, LVEF comparisons by $2 \mathrm{DE}$ and CMR were very close with relatively wide limits of agreement in the Bland-Altman analysis. In a smaller study by Jenkins et al, a quarter of patients with reduced systolic function had $>10 \% \mathrm{EF}$ difference which also corresponds to our finding of relatively wide limits of agreements [22].

Again, both studies have not reported on the influence of infarct location. Our data in a large group of STEMI patients suggest that particularly after non-anterior MI caution is advised when using $2 \mathrm{DE}$ for LVEF measurements. It is not known if this is a phenomenon confined to the acute postinfarct period or if this could play a role after a more distant infarction, as previous studies even with more distant infarction have not looked into this.

An explanation for the influence of infarct location might be that wall motion abnormalities at the apex after anterior infarction are reflected in both single-plane views in 2DE, whereas after inferior infarction wall motion abnormalities are usually only seen in $2 \mathrm{CV}$. Even more problematic is posterior myocardial infarction which is only depicted in $3 \mathrm{CV}$ which is not included in the Simpson method. Furthermore, wall motion abnormalities at the apex are easier to visualise due to the proximity to the ultrasound probe, whereas e.g. the lateral wall can be more difficult to visualise due to artefacts.

In terms of prognosis after STEMI, we demonstrated that both imaging modalities offered similar predictive values for MACE when a LVEF of $<52 \%$ was applied. Several studies have shown that baseline imaging indices were powerfully and independently predictive of all major outcomes when obtained with either 2DE [30, 31] or CMR [32-34]; however, it remains unclear if the predictive values of universally accepted indices (i.e. LVEF) obtained by 2DE and CMR at baseline are comparable. Waha et al have performed both modalities at baseline and showed that several CMR parameters including
LVEF added incremental prognostic value above traditional outcome markers including LVEF by 2DE [16].

Echocardiography requires a thorough knowledge of anatomy and physiology as well as technical skill which can only be gained through supervised education and training in an appropriate environment [35]. It is widely used by a wide range of technicians and physicians, both younger and less experienced on one end, up to extremely experienced examiners with log-books comprising many thousand exams, which will almost certainly make a difference, despite never being formally proven. For this reason, we have put special emphasis on assessing 2DE image quality and have excluded the echocardiograms of the lowest quality. Further, the echocardiograms in this study were reported twice by a very experienced examiner with the appropriate accreditation. In terms of $L V$ volumes, we and others reported an underestimation of about $10 \%$ of the end-diastolic volume by $2 \mathrm{DE}[36,37]$; others however have reported an underestimation of $>30 \%$ $[22,29]$. It is plausible that the degree of underestimation correlates with the degree of LV remodelling after MI. The largest net difference was reported in a patient group with very significant LV remodelling [22]. This degree of LV volume underestimation by $2 \mathrm{DE}$ versus CMR across studies appears to preclude comparability.

\section{Limitations}

Several limitations of our study need to be mentioned. Firstly, a significant number of echocardiograms needed to be excluded upfront in our analysis due to the lack of appropriately stored views and low imaging quality; however, strict quality control is important in an operator-dependent technique like 2D echocardiography. Early measurements of LVEF after STEMI can be misleading because of increase in contractility in uninvolved territories [38-40] and improvement in LVEF may occur in patients who are reperfused [41]. Other factors can influence measurements in the phase of acute cardiac disease, like different loading conditions, stress, arrhythmia or tachycardia. In this study, CMR and 2DE were performed very close to each other (mean 0.4 days) but not truly sequentially; therefore, haemodynamic differences between exams cannot be fully ruled out. However, mean LV function was relatively preserved in our population. Secondly, a majority of patients were receiving beta blockers $(86 \%)$ and only a minority were receiving diuretic therapy $(16 \%)$. These factors as well as the normal mean heart rate during CMR (74) all indicate haemodynamic stability.

Furthermore, due to the small number of patients with severely reduced LVEF, our data cannot be extrapolated to this particularly important subgroup. Pellika et al have recently compared echocardiography with CMR in the STICH Trial, a large trial including patients with previous myocardial infarction with significant left ventricular dysfunction and have 
found only moderate correlation of LVEF by CMR and 2DE, although in $46 \%$ of patients only single-plane echocardiography was performed [42]. Similarly, this applies to other acute cardiomyopathies with regional wall motion abnormalities or healthy probands.

We are unfortunately unable to offer data about myocardial strain in our patients, as assessed by myocardial tagging or CMR feature tracking [43]. Myocardial strain imaging is a promising tool for better quantification of global and regional left ventricular functions in a broad range of cardiovascular diseases, including myocardial infarction or myocarditis [44-46]. Several CMR methods have been described and there is growing evidence that these techniques offer robust information after STEMI [47]. However, the improvement of risk stratification beyond traditional CMR indexes such as left ventricular ejection fraction remains controversial. Recent clinical outcome studies in STEMI patients using feature tracking or displacement encoding with stimulated echoes (DENSE) CMR indicated a strong prognostic role of global longitudinal strain or circumferential strain [48-50].

Despite these factors, our data suggest that LVEF measurements by $2 \mathrm{DE}$ at baseline after STEMI are generally robust provided adequate image and reporting quality and the infarct location are taken into consideration.

In summary, 2D echocardiography significantly underestimated LVEF in comparison with CMR at baseline after non-anterior STEMI, whereas after anterior infarction, measurements were within acceptable limits of agreement. Despite these differences, a reduced LVEF of $<52 \%$ by both imaging modalities had similar prognostic values for MACE over a mean follow-up of 41 months. Further study is warranted to investigate if this phenomenon is confined to the acute post-infarct period as it would have significant impact on further device therapy.

Funding Open access funding provided by University of Innsbruck and Medical University of Innsbruck. The authors state that this work has not received any funding.

\section{Compliance with ethical standards}

Guarantor The scientific guarantor of this publication is Gert Klug.

Conflict of interest The authors of this manuscript declare no relationships with any companies, whose products or services may be related to the subject matter of the article.

Statistics and biometry No complex statistical methods were necessary for this paper.

Informed consent Written informed consent was obtained from all subjects (patients) in this study.

Ethical approval Institutional Review Board approval was obtained.
Study subjects or cohorts overlap The patients in this study were participants in a prospective analysis enrolling patients who underwent CMR after acute STEMI treated with primary percutaneous coronary intervention (PCI). Multiple studies have been published so far from this group, but this is the first study that has systematically reported on comparing aspects on imaging with 2D echocardiography versus cardiac MRI in the acute period after myocardial infarction.

\section{Methodology \\ - prospective \\ - observational \\ - performed at one institution}

Open Access This article is distributed under the terms of the Creative Commons Attribution 4.0 International License (http:// creativecommons.org/licenses/by/4.0/), which permits unrestricted use, distribution, and reproduction in any medium, provided you give appropriate credit to the original author(s) and the source, provide a link to the Creative Commons license, and indicate if changes were made.

\section{References}

1. Burns RJ, Gibbons RJ, Yi Q et al (2002) The relationships of left ventricular ejection fraction, end-systolic volume index and infarct size to six-month mortality after hospital discharge following myocardial infarction treated by thrombolysis. J Am Coll Cardiol 39:30-36

2. Reinstadler SJ, Thiele H, Eitel I (2015) Risk stratification by cardiac magnetic resonance imaging after ST-elevation myocardial infarction. Curr Opin Cardiol 30:681-689

3. Moller JE, Hillis GS, Oh JK, Reeder GS, Gersh BJ, Pellikka PA (2006) Wall motion score index and ejection fraction for risk stratification after acute myocardial infarction. Am Heart J 151:419 425

4. Reindl M, Reinstadler SJ, Tiller C et al (2018) ACEF score adapted to ST-elevation myocardial infarction patients: the ACEF-STEMI score. Int J Cardiol 264:18-24

5. Ibanez B, James S, Agewall S et al (2018) 2017 ESC guidelines for the management of acute myocardial infarction in patients presenting with ST-segment elevation: the task force for the management of acute myocardial infarction in patients presenting with STsegment elevation of the European Society of Cardiology (ESC). Eur Heart J 39:119-177

6. Chen X, Liu F, Xu H et al (2017) Left ventricular diastolic dysfunction in patients with ST-elevation myocardial infarction following early and late reperfusion by coronary intervention. Int J Cardiol 228:886-889

7. Malik SB, Chen N, Parker RA III, Hsu JY (2017) Transthoracic echocardiography: pitfalls and limitations as delineated at cardiac CT and MR imaging. Radiographics 37:383-406

8. Thomson HL, Basmadjian AJ, Rainbird AJ et al (2001) Contrast echocardiography improves the accuracy and reproducibility of left ventricular remodeling measurements: a prospective, randomly assigned, blinded study. J Am Coll Cardiol 38:867-875

9. Lancellotti P, Price S, Edvardsen T et al (2015) The use of echocardiography in acute cardiovascular care: recommendations of the European Association of Cardiovascular Imaging and the Acute Cardiovascular Care Association. Eur Heart J Cardiovasc Imaging 16:119-146

10. Thorstensen A, Dalen H, Amundsen BH, Aase SA, Stoylen A (2010) Reproducibility in echocardiographic assessment of the left ventricular global and regional function, the HUNT study. Eur J Echocardiogr 11:149-156 
11. Thavendiranathan P, Grant AD, Negishi T, Plana JC, Popovic ZB, Marwick TH (2013) Reproducibility of echocardiographic techniques for sequential assessment of left ventricular ejection fraction and volumes: application to patients undergoing cancer chemotherapy. J Am Coll Cardiol 61:77-84

12. Reindl M, Reinstadler SJ, Tiller C et al (2019) Prognosis-based definition of left ventricular remodeling after ST-elevation myocardial infarction. Eur Radiol 29:2330-2339

13. Klug G, Metzler B (2013) Assessing myocardial recovery following ST-segment elevation myocardial infarction: short- and longterm perspectives using cardiovascular magnetic resonance. Expert Rev Cardiovasc Ther 11:203-219

14. Guaricci AI, Carrabba N, Aquaro GD et al (2019) Advanced imaging techniques (CT and MR): gender-based diagnostic work-up in ischemic heart disease? Int J Cardiol 286:234-238

15. Nowosielski M, Schocke M, Mayr A et al (2009) Comparison of wall thickening and ejection fraction by cardiovascular magnetic resonance and echocardiography in acute myocardial infarction. $\mathrm{J}$ Cardiovasc Magn Reson 11:22

16. de WS, Eitel I, Desch S et al (2014) Prognosis after ST-elevation myocardial infarction: a study on cardiac magnetic resonance imaging versus clinical routine. Trials 15:249

17. Reindl M, Reinstadler SJ, Feistritzer HJ et al (2017) Persistent Twave inversion predicts myocardial damage after ST-elevation myocardial infarction. Int J Cardiol 241:76-82

18. Reinstadler SJ, Feistritzer HJ, Klug G et al (2016) High-sensitivity troponin $\mathrm{T}$ for prediction of left ventricular function and infarct size one year following ST-elevation myocardial infarction. Int J Cardiol 202:188-193

19. Thygesen K, Alpert JS, Jaffe AS et al (2012) Third universal definition of myocardial infarction. Eur Heart J 33:2551-2567

20. Reindl M, Feistritzer HJ, Reinstadler SJ et al (2018) Thyroidstimulating hormone and adverse left ventricular remodeling following ST-segment elevation myocardial infarction. Eur Heart J Acute Cardiovasc Care Apr 1:1-10

21. Lang RM, Badano LP, Mor-Avi V et al (2015) Recommendations for cardiac chamber quantification by echocardiography in adults: an update from the American Society of Echocardiography and the European Association of Cardiovascular Imaging. Eur Heart J Cardiovasc Imaging 16:233-270

22. Jenkins C, Moir S, Chan J, Rakhit D, Haluska B, Marwick TH (2009) Left ventricular volume measurement with echocardiography: a comparison of left ventricular opacification, threedimensional echocardiography, or both with magnetic resonance imaging. Eur Heart J 30:98-106

23. Klug G, Mayr A, Schenk S et al (2012) Prognostic value at 5 years of microvascular obstruction after acute myocardial infarction assessed by cardiovascular magnetic resonance. J Cardiovasc Magn Reson 14:46

24. Klug G, Trieb T, Schocke M et al (2009) Quantification of regional functional improvement of infarcted myocardium after primary PTCA by contrast-enhanced magnetic resonance imaging. J Magn Reson Imaging 29:298-304

25. Beek AM, Kuhl HP, Bondarenko O et al (2003) Delayed contrastenhanced magnetic resonance imaging for the prediction of regional functional improvement after acute myocardial infarction. J Am Coll Cardiol 42:895-901

26. Bondarenko O, Beek AM, Hofman MB et al (2005) Standardizing the definition of hyperenhancement in the quantitative assessment of infarct size and myocardial viability using delayed contrastenhanced CMR. J Cardiovasc Magn Reson 7:481-485

27. Reinstadler SJ, Klug G, Feistritzer HJ et al (2016) Prognostic value of left ventricular global function index in patients after ST-segment elevation myocardial infarction. Eur Heart J Cardiovasc Imaging 17:169-176
28. Klug G, Reinstadler SJ, Feistritzer HJ et al (2016) Cardiac index after acute ST-segment elevation myocardial infarction measured with phase-contrast cardiac magnetic resonance imaging. Eur Radiol 26:1999-2008

29. Mistry N, Halvorsen S, Hoffmann P et al (2010) Assessment of left ventricular function with magnetic resonance imaging vs. echocardiography, contrast echocardiography, and single-photon emission computed tomography in patients with recent ST-elevation myocardial infarction. Eur J Echocardiogr 11:793-800

30. Solomon SD, Skali H, Anavekar NS et al (2005) Changes in ventricular size and function in patients treated with valsartan, captopril, or both after myocardial infarction. Circulation 111:3411-3419

31. Nicolosi GL, Latini R, Marino P et al (1996) The prognostic value of predischarge quantitative two-dimensional echocardiographic measurements and the effects of early lisinopril treatment on left ventricular structure and function after acute myocardial infarction in the GISSI-3 trial. Gruppo Italiano per lo Studio della Sopravvivenza nell'Infarto Miocardico. Eur Heart J 17:1646-1656

32. Symons R, Pontone G, Schwitter J et al (2018) Long-term incremental prognostic value of cardiovascular magnetic resonance after ST-segment elevation myocardial infarction: a study of the collaborative registry on CMR in STEMI. JACC Cardiovasc Imaging 11: 813-825

33. Bodi V, Sanchis J, Nunez J et al (2009) Prognostic value of a comprehensive cardiac magnetic resonance assessment soon after a first ST-segment elevation myocardial infarction. JACC Cardiovasc Imaging 2:835-842

34. Larose E, Rodes-Cabau J, Pibarot P et al (2010) Predicting late myocardial recovery and outcomes in the early hours of STsegment elevation myocardial infarction traditional measures compared with microvascular obstruction, salvaged myocardium, and necrosis characteristics by cardiovascular magnetic resonance. J Am Coll Cardiol 55:2459-2469

35. Popescu BA, Andrade MJ, Badano LP et al (2009) European Association of Echocardiography recommendations for training, competence, and quality improvement in echocardiography. Eur J Echocardiogr 10:893-905

36. Chuang ML, Hibberd MG, Salton CJ et al (2000) Importance of imaging method over imaging modality in noninvasive determination of left ventricular volumes and ejection fraction: assessment by two- and three-dimensional echocardiography and magnetic resonance imaging. J Am Coll Cardiol 35:477-484

37. Saloux E, Labombarda F, Pellissier A et al (2014) Diagnostic value of three-dimensional contrast-enhanced echocardiography for left ventricular volume and ejection fraction measurement in patients with poor acoustic windows: a comparison of echocardiography and magnetic resonance imaging. J Am Soc Echocardiogr 27: $1029-1040$

38. Rechavia E, de SR, Nihoyannopoulos P, Lammertsma AA, Jones T, Maseri A (1995) Hyperdynamic performance of remote myocardium in acute infarction. Correlation between regional contractile function and myocardial perfusion. Eur Heart J 16:1845-1850

39. Feldman RL, Macdonald RG, Nichols WW, Conti CR, Pepine CJ (1984) Effects of acute coronary occlusion on hemodynamics in an adjacent coronary artery in dogs. Am J Cardiol 54:1103-1107

40. Gascho JA, Beller GA (1987) Adverse effects of circumflex coronary artery occlusion on blood flow to remote myocardium supplied by a stenosed left anterior descending coronary artery in anesthetized open-chest dogs. Am Heart J 113:679-683

41. Sheehan FH, Doerr R, Schmidt WG et al (1988) Early recovery of left ventricular function after thrombolytic therapy for acute myocardial infarction: an important determinant of survival. J Am Coll Cardiol 12:289-300

42. Pellikka PA, She L, Holly TA et al (2018) Variability in ejection fraction measured by echocardiography, gated single-photon emission computed tomography, and cardiac magnetic resonance in 
patients with coronary artery disease and left ventricular dysfunction. JAMA Netw Open 1:e181456

43. Schuster A, Hor KN, Kowallick JT, Beerbaum P, Kutty S (2016) Cardiovascular magnetic resonance myocardial feature tracking: concepts and clinical applications. Circ Cardiovasc Imaging 9: e004077

44. Khan JN, Singh A, Nazir SA, Kanagala P, Gershlick AH, McCann GP (2015) Comparison of cardiovascular magnetic resonance feature tracking and tagging for the assessment of left ventricular systolic strain in acute myocardial infarction. Eur J Radiol 84:840-848

45. Doerner J, Bunck AC, Michels G, Maintz D, Baessler B (2018) Incremental value of cardiovascular magnetic resonance feature tracking derived atrial and ventricular strain parameters in a comprehensive approach for the diagnosis of acute myocarditis. Eur $\mathrm{J}$ Radiol 104:120-128

46. Schmidt B, Dick A, Treutlein M et al (2017) Intra- and interobserver reproducibility of global and regional magnetic resonance feature tracking derived strain parameters of the left and right ventricle. Eur J Radiol 89:97-105

47. Mangion K, McComb C, Auger DA, Epstein FH, Berry C (2017) Magnetic resonance imaging of myocardial strain after acute ST- segment-elevation myocardial infarction: a systematic review. Circ Cardiovasc Imaging Aug;10(8)

48. Gavara J, Rodriguez-Palomares JF, Valente F et al (2018) Prognostic value of strain by tissue tracking cardiac magnetic resonance after ST-segment elevation myocardial infarction. JACC Cardiovasc Imaging 11:1448-1457

49. Eitel I, Stiermaier T, Lange T et al (2018) Cardiac magnetic resonance myocardial feature tracking for optimized prediction of cardiovascular events following myocardial infarction. JACC Cardiovasc Imaging 11:1433-1444

50. Mangion K, Carrick D, Carberry J et al (2019) Circumferential strain predicts major adverse cardiovascular events following an acute ST-segment-elevation myocardial infarction. Radiology 290:329-337

Publisher's note Springer Nature remains neutral with regard to jurisdictional claims in published maps and institutional affiliations. 